\title{
The Shear Bond Strengths of Composite Bonded to Dentine Following Treatment with Two Dentine Occluding Desensitizing Toothpastes
}

\author{
Shivaughn Marchan ${ }^{*}$, Daniel White ${ }^{2}$, Jessica Pruszynski ${ }^{3}$, Tichard Manwah ${ }^{4}$, \\ Vishala Bassaw ${ }^{4}$, William Smith1 \\ ${ }^{1}$ Unit of Restorative Dentistry, School of Dentistry, Faculty of Medical Sciences, The University of the \\ West Indies, St. Augustine, Trinidad and Tobago \\ ${ }^{2}$ The University of Trinidad and Tobago, Point Lisas Campus, Point Lisas, Couva, Trinidad and Tobago \\ ${ }^{3}$ Division of Biostatistics, Medical College of Wisconsin, Milwaukee, USA \\ ${ }^{4}$ School of Dentistry, Faculty of Medical Sciences, The University of the West Indies, St. Augustine, \\ Trinidad and Tobago

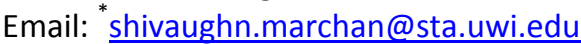

Received 12 January 2014; revised 17 February 2014; accepted 26 February 2014

Copyright (C) 2014 by authors and Scientific Research Publishing Inc.

This work is licensed under the Creative Commons Attribution International License (CC BY). http://creativecommons.org/licenses/by/4.0/

(c) (i) Open Access

\section{Abstract}

Aim: This study determined whether prior brushing with desensitizing toothpastes (Sensodyne Rapid Relief and Colgate Pro-Relief) affected the shear bond strengths of composite to dentine surfaces after appropriate use of two different dentine bonding agents (SingleBond and PQ-1). Materials and Methods: Sixty caries free molar teeth were cleaned, disinfected and embedded in individual cylinders of polymethylmethracyrlate. The occulsal surfaces were flattened to expose dentine and finished down with $\mathbf{6 0 0}$ grit silicone carbide paper. The teeth were randomly divided into six groups of 10 teeth each. Two groups served as control where dentine surfaces were brushed with pumice slurry. Of the remaining 4 groups, 2 groups were brushed with Sensodyne Rapid Relief and 2 groups brushed with Colgate Pro-Relief. The dentine surfaces were brushed to simulate 2 weeks of twice-daily toothpaste use. The specimens in each of the control groups and experimental groups were subjected to dentine bonding procedures (Single Bond or PQ-1), and application of hybrid composite resin (Z-250) according to the manufacturer's instructions. A universal material testing machine, with a cross head speed of $0.05 \mathrm{~mm} / \mathrm{min}$ was used to determine shear bond strengths. Results: Mean shear bond strengths ranged from 5.71 MPa (Colgate pretreatment, composite bonded with Single Bond) to 9.07 MPa (Sensodyne pretreatment, com-

*Corresponding author.

How to cite this paper: Marchan, S., et al. (2014) The Shear Bond Strengths of Composite Bonded to Dentine Following Treatment with Two Dentine Occluding Desensitizing Toothpastes. Open Journal of Stomatology, 4, 121-125. 
posite bonded with Single Bond). Results showed neither of the main effects, of toothpaste type or bonding agent were significant $(p>0.05)$ but their interaction was $(F=4.25, p=0.02)$. Post hoc analysis showed that teeth brushed with the Sensodyne group, treated with the unfilled dentine bonding agent had a significantly higher bond strength than those treated with the filled dentine bonding agent. Conclusion: The desensitizing toothpastes that use the mechanism of occlusion of open dentinal tubules in the preliminary management of tooth sensitivity should not have an adverse effect on future restorative treatment modalities.

\section{Keywords}

\section{Shear Bond Strengths; Desensitizing Toothpastes}

\section{Introduction}

Tooth surface loss is a common dental problem, which can be attributed to various etiologies such as attrition, abfraction, abrasion and erosion [1]. Rafeek et al. reported a prevalence rate of $72 \%$ for all types of wear and severity (mild, moderate and severe) whilst Smith et al. quoted prevalence rates of $62.2 \%$ for wear at the cervical aspect of teeth [2] [3]. The most common and earliest presenting symptom of tooth surface loss is dentine sensitivity due to exposed and patent dentinal tubules, for which there are a number of management modalities. Initial management normally involves conservative modalities such as prescription of desensitizing toothpastes. The mechanism of action of desensitizing toothpastes is either one of disruption of neural pathways in the transmission of pain or physical occlusion of dentinal tubules [3]-[5].

The use of arginine, a naturally occurring salivary amino acid and calcium carbonate containing toothpastes, has been reported to provide immediate and lasting relief to dentine sensitivity as it protects the dentinal tubules from stimuli [5]. This is achieved by the interaction between arginine and calcium carbonate which leads to the formation of a conterminous, paste-like calcium compound that adheres to dentinal tubules and seals them [5]. Alternatively toothpaste based on $8 \%$ strontium acetate salts is also known to occlude patent dentinal tubules. Parkinson et al. demonstrated that strontium acetate derived dentine plugs may be visualized deeper within the tubule and are more resistant to the constant acidic challenges occurring within the oral cavity when compared with its arginine counterpart [6].

If such management strategies are ineffective the next phase of treatment may be the use of restorative means where dental adhesives and/or composite restorations are employed to physically occlude patent dentinal tubules and protect exposed dentine surfaces from external stimuli. Reliable bonding depends on the removal or modification of the smear layer and associated dentine plugs, increasing the surface energy of dentine and infiltration of resin adhesive into dentine tubules and between inter-fibrillar collagen spaces [7] [8]. Theoretically the presence of dentine plug precipitates formed as result of prior toothpaste use, may resist the etching potential of phosphoric acid. This in turn may affect the strength of the bond between dentine and the overlying adhesive/ composite complex.

The aim of this study is to determine whether brushing with desensitizing toothpastes with a mechanism of action of occlusion of dentinal tubules can affect the shear bond strengths of composite to dentine surfaces after appropriate use of two different fifth generation bonding agents.

\section{Materials and Methods}

Sixty caries free, un-restored, periodontally involved extracted posterior human teeth were obtained and stored according to ISO Specification 11405 (Testing of adhesion to tooth structure) [9]. The roots of the teeth were cleaned of blood and obvious soft tissue using running water. Calculus was removed using powered scaler tips (FS1-100, Cavitron, Dentsply, York, PA, USA) in a magnetostrictive ultrasonic device (Cavitron Bobcat, Dentsply, York, PA, USA).

The teeth were embedded in individual cylinders of polymethylmethracyrlate (Acratray, Henry Schein, Melville, NY, USA). The occlusal surface of each tooth was ground flat using a water cooled model trimmer (Model 40,217, Whip Mix Corp, Louisville, KY, USA) to create a flattened dentine surface in the occlusal third of the 
tooth, with care being taken not to expose pulpal anatomy. Teeth were then planed against wet silicon carbide paper of grit size of P600 (Eagle Abrasives Inc, Norcross, GA, USA), of a median grain size of ( $25.8 \pm 1 \mu \mathrm{m})$ in accordance with ISO specification 6344-1:1998. The specimens were returned to distilled water at $4^{\circ} \mathrm{C}$ for two weeks prior to the brushing regimen.

The teeth were randomly divided into three groups of 20 teeth each: 1) control group; 2) Sensodyne Rapid Relief Group; 3) Colgate Pro-relief group.

The control groups were brushed with flour pumice using disposable prophylaxis brushes in a slow speed contra-angle handpiece whilst the experimental groups were brushed with a pea sized amount of either the 8\% arginine calcium carbonate toothpaste (Colgate Pro-Relief, Colgate Palmolive Company, Park Ave, New York City, New York, USA) or the 8\% strontium acetate toothpaste (Sensodyne Rapid Relief, GlaxoSmithKlein, Brentford, United Kingdom) for a total of 3 minutes four times a day for 7 days to simulate 14 days of toothpaste use twice daily. The specimens were returned to distilled water storage for 1 week prior to the bonding procedure.

The teeth of the control and experimental groups were further subdivided into 2 groups, to give a total of 6 groups of 10 specimens each, whose brushed dentine surfaces would be treated with either a filled dentinebonding agent (Perma Quick 1, PQ-1 Ultradent Products Inc, South Jordan, UT, USA) or an unfilled dentine bonding agent (Single Bond, 3M ESPE, St Paul, MN, USA). The dentine surfaces were etched with 37\% phosphoric acid etchant for 15 seconds and then thoroughly rinsed with distilled water and gently dried using oil free compressed air to reveal frosty surfaces. The respective dentine-bonding agents were applied using microtips and gently thinned with compressed air and light cured (Elipar Freelight 2, 3M ESPE, St Paul, MN, USA) for 10 seconds. A transparent plastic mold of internal diameter $6 \mathrm{~mm}$ was stabilized in the middle of the dentine surface. A $2 \mathrm{~mm}$ increment of composite (Z250 Composite Shade A3, 3M ESPE, St Paul, MN, USA) was placed in the mold and light cured for 40 seconds by using a sweeping action around the transparent mold. The specimens were returned to fresh distilled water at room temperature for storage for a period of two weeks prior to mechanical testing.

\section{Shear Bond Testing}

A universal material testing machine (Hounsfield H50KS Tensometer, Tinius Olsen Ltd, Redhill, Surrey, UK) was used to determine shear bond strengths. Each specimen (Tooth-Composite cylinder in its embedding resin) was placed in a specialized mounting jig that facilitated placement in the Tensometer. A shear probe, of machined stainless steel, with circular cross-section and a sharp counter-bevel was utilized to facilitate shear testing. The counter-bevel acted as a knife-edge probe that allowed direct contact with the specimen at the tooth-composite interface. Following removal from distilled water, the shear force was applied with a load cell of $500 \mathrm{~N}$ to the interface at a crosshead speed of $0.5 \mathrm{~mm} / \mathrm{min}$ until failure occurred. The peak load at failure was converted to shear force (MPa) by diving the numerical value obtained for force $(\mathrm{N})$ by the cross sectional area of the cylindrical composite specimen $\left(\pi \mathrm{Xr}^{2}\right.$ where $\mathrm{r}$ is the radius of the cylindrical specimen).

Data were analyzed using SAS software version 9.3. The means and standard deviations were calculated for each group. The two factor ANOVA was used to assess differences in bond strength. Included factors in the model were toothpaste type, bonding agent and their interaction.

\section{Results}

Removal of the transparent mold from around a composite cylinder on a tooth treated with Sensodyne Rapid Relief and bonded with SingleBond resulted in one specimen debonding from the dentine substrate prior to shear testing. This failed specimen was not subjected to statistical analysis.

Mean shear bond strengths ranged from 5.71 MPa (Colgate pretreatment, composite bonded with SingleBond) to 9.07 MPa (Sensodyne pretreatment, composite bonded with SingleBond). The Means and standard deviations are presented in Table 1.

The use of the homogeneity-of-variance Levene's test demonstrated that the specimens were selected from a normal population with equal variances.

Results showed neither of the main effects were significant $(\mathrm{p}>0.05)$ but their interaction was $(\mathrm{F}=4.25, \mathrm{p}=$ 0.02). Post hoc analysis showed that teeth within the Sensodyne group, and treated with the unfilled dentine bonding agent had a significantly higher bond strength than those treated with the filled dentine bonding agent. 
Table 1. Mean shear bond strengths (MPa) and standard deviations of composite bonded to dentine using two bonding agents following treatment with pumice (control), Colgate Pro-Relief and Sensodyne Rapid Relief.

\begin{tabular}{cccc}
\hline Toothpaste & Dentine bonding agent & n & Mean (SD) \\
\hline Pumice & SingleBond & 10 & $7.09(1.90)$ \\
& PQ1 & 10 & $7.12(2.86)$ \\
Colgate pro-relief & SingleBond & 10 & $5.71(2.25)$ \\
& PQ1 & 10 & $7.27(2.40)$ \\
Sensodyne rapid relief & SingleBond & 9 & $9.07(3.17)$ \\
& PQ1 & 10 & $5.82(2.98)$ \\
\hline
\end{tabular}

\section{Discussion}

There are two main methods to determine bond strengths of composite to dentine substrates-tests of shear bond strengths or tensile bond strengths, specifically micro-tensile bond strengths. Due to the strict prerequisite of alignment of specimens within the testing apparatus for tests of tensile bonding and to avoid uneven stress distribution during loading, the authors decided to use a shear test method to determine bond strengths in this particular study [9]. Tests of shear bond strengths require exacting positioning of the shearing blade at the interface between the composite and underlying dentine substrate, which was easily controlled due to the configuration of the test apparatus and shearing probe in this particular study [9].

When considering Colgate ProRelief, this study demonstrated no statistically significant differences in shear bond strengths to composite after 2 weeks of simulated brushing between the control and experimental toothpaste groups. Furthermore the shear bond of composite to dentine when bonded with either of the dentine bonding agents (Single Bond or PQ1) was not affected by neither the $8 \%$ arginine/calcium carbonate nor the 8\% strontium acetate toothpastes. The concurs with the findings of Canares et al., who demonstrated that pre-treatment with desensitizing toothpastes containing $8 \%$ arginine and calcium carbonate had no effect on the bond strength of composites to dentine compared to controls where pumice was the pre-treatment [10]. This was an unexpected result as any toothpaste that forms precipitates on dentine surfaces could theoretically affect hybridization. It can be inferred that use of phosphoric acid in smear layer removal also resulted in the dissolution of any dentine precipitates formed as a result of toothpaste use. An alternative explanation may be that the precipitates formed on the surface of the dentine substrate, over the period of storage are not tenacious enough to affect formation of a hybrid layer. These theories would have to be further investigated using dentine disk models and scanning electron microscopy, to visualize not only the state of dentine surfaces after etching with phosphoric acid but also the interaction of both filled and unfilled dentine bonding agents with precipitates of such toothpastes on dentine surfaces and within the tubular structures.

With respect to the Sensodyne experimental group, statistical analysis revealed that the interaction between the toothpaste and the bonding agent produced statistically higher bond strengths for the Single Bond (unfilled) group compared to the Perma Quick (filled) group. Whilst filled adhesives are purported to reinforce the hybrid zone, Ateyah et al. have shown that they have lower shear bond strengths compared with their unfilled counterparts [11]. This however does not fully explain the observed data. A dentine disc model that could visualize the interaction between different types of adhesives and strontium acetate precipitates would be germane to improve our understanding of the current results.

It is noteworthy to point out that the absolute mean values, which ranged from 5.71 to $9.07 \mathrm{MPa}$, for shear bond strengths obtained were much lower numerically compared to a similar study [10]. Whilst similar studies cannot be directly compared due to slight variations in methodology the shear bond values achieved are some cause for concern. Canares et al. performed tests of shear bond strength at 48 hours following bonding of the specimens whilst this current study stored the specimens for two weeks prior to testing. There may have been some hydrolytic deterioration of the bond during the period of storage. Further testing would have to compare bond strengths 24 - 48 hours following the bonding procedure compared to longer periods of storage.

The results of the present study show that either desensitizing toothpaste that uses the mechanism of occlusion of open dentinal tubules in the preliminary management of tooth sensitivity should not have an adverse effect on future restorative treatment modalities. 


\section{Acknowledgements}

We wish to thank The School of Dentistry, Faculty of Medical Sciences, The University of the West Indies for funding this research. This research was presented at the annual Student Research Day conference, Faculty of Medical Sciences, The University of the West Indies.

\section{References}

[1] Rafeek, R., Marchan, S., Eder, A. and Smith, W.A.J. (2006) Tooth Surface Loss in Adult Subjects Attending a University Dental Clinic in Trinidad. International Dental Journal, 56, 181-186.

[2] Smith, W.A.J., Marchan, S. and Rafeek, R. (2008) The Prevalence and Severity of Non-Carious Cervical Lesions in a Group of Patients Attending a University Hospital in Trinidad. Journal of Oral Rehabilitation, 35, 128-134.

[3] Salvato, A.R., Clart, G.E., Gingold, J. and Curro, F.A. (1992) Clinical Effectiveness of a Dentifrice Containing Potassium Chloride as a Desensitizing Agent. American Journal of Dentistry, 5, 303-306.

[4] Silverman, G., Gingold, J. and Curro, F.A. (1994) Desensitizing Effect of a Potassium Chloride Dentifrice. American Journal of Dentistry, 7, 9-12.

[5] Petrou, I., Heu, R., Stranick, M., Lavender, S., Zaidel, L., Cummins, D., Sullivan, R.J., Hseuh, C. and Gimzewski, J.K. (2009) A Breakthrough Therapy for Dentin Hypersensitivity: How Dental Products Containing 8\% Arginine and Calcium Carbonate Work to Deliver Effective Relief of Sensitive Teeth. Journal of Clinical Dentistry, 20, 23-31.

[6] Parkinson, C.R. and Willson, R.J. (2011) An in Vitro Investigation of Two Currently Marketed Dentin Tubule Occlusion Dentifrices. Journal of Clinical Dentistry, 22, 6-10.

[7] Pashley, D.H., Ciucchi, B. and Sano, H. (1994) Dentin as a Bonding Substrate. Deutsche Zahnarztl Zeitschrift, 49, 760-763.

[8] Pashley, D.H., Ciucchi, B., Sano, H. and Horner, J.A. (1993) Permeability of Dentin to Adhesive Resins. Quintessence International, 24, 618-631.

[9] ISO Specification ISO/TS 11405 (E) (2003) Dental Materials-Testing of Adhesion to Tooth Structure.

[10] Canares, G., Salgado, T., Pines, M.S. and Wolff, M.S. (2012) Effect of an 8\% Arginine and Calcium Carbonate Desensitizing Toothpaste on Shear Dentine Bond Strength. Journal of Clinical Dentistry, 23, 68-70.

[11] Ateyah, N.Z. and Elhejazi, A.A. (2004) Shear Bond Strengths and Microleakage of Four Types of Dentine Adhesive Materials. Journal of Contemporary Dental Practice, 5, 63-73. 